\title{
sPIF promotes myoblast differentiation and utrophin expression while inhibiting fibrosis in Duchenne muscular dystrophy via the H19/miR-675/let-7 and miR-21 pathways
}

Daria Morgoulis ${ }^{1}$, Peter Berenstein ${ }^{1}$, Simona Cazacu², Gila Kazimirsky ${ }^{1}$, Amir Dori ${ }^{3}$, Eytan R. Barnea ${ }^{4}$ and Chaya Brodie ${ }^{1,2}$

\begin{abstract}
Duchenne muscular dystrophy (DMD) is a progressive, lethal, X-linked disease of skeletal and cardiac muscles caused by mutations in the dystrophin gene. Loss of dystrophin leads to muscle fiber damage and impairment of satellite cell asymmetric division, which are essential for muscle regeneration. These processes ultimately result in muscle wasting and the replacement of the degenerating muscles by fibrogenic cells, a process that leads to the generation of fibrotic tissues. Preimplantation factor (PIF) is an evolutionary conserved 15-amino acid peptide secreted by viable mammalian embryos. Synthetic PIF (sPIF) reproduces the protective/regenerative effects of the endogenous peptide in immune disorders and transplantation models. In this study, we demonstrated that sPIF treatment promoted mouse and human myoblast differentiation and inhibited the expression of collagen 1A1, collagen $1 \mathrm{~A} 2$, and TGF- $\beta$ in DMD patient-derived myoblasts. Additionally, sPIF increased the expression of utrophin, a homolog of dystrophin protein. sPIF effects were mediated via the upregulation of IncRNA H19 and miR-675 and downregulation of let-7. sPIF also inhibited the expression of miR-21, a major fibrosis regulator. The administration of sPIF in mdx mice significantly decreased serum creatine kinase and collagen I and collagen IV expression in the diaphragm, whereas it increased utrophin expression in the diaphragm, heart and quadriceps muscles. In conclusion, sPIF promoted the differentiation of DMD myoblasts, increased utrophin expression via the H19/miRNA-675/let-7 pathway, and reduced muscle fibrosis possibly via the upregulation of miR-675 and inhibition of miR-21 expression. These findings strongly support pursuing SPIF as a potential therapeutic agent for DMD. Moreover, the completion of an sPIF phase I safety trial will further promote the use of sPIF for the treatment of muscular dystrophies.
\end{abstract}

\section{Introduction}

Duchenne muscular dystrophy (DMD) is a recessive, fatal, X-linked disease and the most common form of

\footnotetext{
Correspondence: Chaya Brodie (chaya@brodienet.com)

${ }^{1}$ The Mina and Everard Goodman Faculty of Life Sciences, Bar-llan University, Ramat-Gan, Israel

${ }^{2}$ Department of Neurosurgery, Henry Ford Hospital, Detroit, MI, USA

Full list of author information is available at the end of the article.

These authors contributed equally: Amir Dori, Eytan R. Barnea and Chaya Brodie Edited by A. Oberst
}

muscular dystrophy ${ }^{1}$. DMD is caused by mutations in the dystrophin gene that can be either spontaneous or inherited $^{2,3}$. The dystrophin protein plays a critical role in the maintenance, integrity, and normal functions of muscle cells, and its loss leads to progressive muscle degeneration ${ }^{4,5}$. Under normal conditions, muscle damage activates quiescent muscle stem cells (satellite cells), which eventually differentiate into mature muscle cells and contribute to tissue regeneration ${ }^{6}$. Recent studies indicate that

\section{(c) The Author(s) 2019}

(c) (i) Open Access This article is licensed under a Creative Commons Attribution 4.0 International License, which permits use, sharing, adaptation, distribution and reproduction in any medium or format, as long as you give appropriate credit to the original author(s) and the source, provide a link to the Creative Commons license, and indicate if changes were made. The images or other third party material in this article are included in the article's Creative Commons license, unless indicated otherwise in a credit line to the material. If material is not included in the article's Creative Commons license and your intended use is not permitted by statutory regulation or exceeds the permitted use, you will need to obtain permission directly from the copyright holder. To view a copy of this license, visit http://creativecommons.org/licenses/by/4.0/. 
dystrophin is also essential for the asymmetric division of satellite cells, and the lack of functional protein interferes with the regenerative capacity of these cells ${ }^{7}$. Thus, muscle regeneration is impaired in DMD, resulting in the replacement of degenerating muscle fibers by fibrotic and fat tissues $^{1,8}$.

Various therapeutic approaches have been explored for treating DMD, including promotion of muscle regeneration $^{9}$, anti-inflammatory and anti-fibrotic agents ${ }^{2,10,11}$, and exon-skipping with antisense oligonucleotides ${ }^{12,13}$. Despite these efforts, there is currently no cure for DMD, and treatments focus mainly on relieving the symptoms and minimizing complications ${ }^{14}$.

Preimplantation factor (PIF) is a 15 -amino acid peptide secreted by viable embryos, which through autotrophic action promotes development and protects against embryo demise due to adverse environments ${ }^{15-18}$. PIF promotes implantation and placental engraftment, and reduces spontaneous and Lipopolysaccharide (LPS)induced fetal demise in an immune intact murine model ${ }^{19-21}$. The synthetic PIF analog (sPIF) replicates the native peptide actions and has comprehensive immune protective and regenerative properties ${ }^{22-24}$. sPIF targets innate immunity (macrophages/neutrophils) and affects activated $\mathrm{T}$-cell proliferation and mixed lymphocyte reaction ${ }^{22}$ as well as decreased NK cytotoxicity $^{25}$. Through an integrated local and systemic effect, sPIF protects against juvenile diabetes ${ }^{26}$, cardiovascular inflammation ${ }^{27}$, radiation-induced injury ${ }^{28}$, neuroinflammation, and neurotrauma in various animal models $^{29-32}$. In addition, sPIF promotes ovarian allotransplantation in transplantation models in primates and semi and allogeneic bone marrow transplantation ${ }^{33,34}$. Recently, sPIF was the subject of a successful Universityinitiated FDA Fast-Track-awarded Phase I clinical trial for autoimmune hepatitis and obtained Orphan Drug Designation (www.clinicaltrials.gov, NCT 02239562).

In this study, we demonstrated that sPIF increased muscle cell differentiation and utrophin level while inhibiting fibrogenic gene expression and identified the H19/ miR-675/let-7 and miR-21 pathways as mediators of sPIF effects. Moreover, sPIF administration to $\mathrm{mdx}$ mice decreased creatine kinase (CK) levels and tissue fibrosis and increased the expression of utrophin, thus inducing a change in biomarkers that suggests a therapeutic effect.

\section{Results}

\section{sPIF increases the differentiation of human and mouse} myoblasts and decreases collagen and TGF- $\beta$ expression

To study the effects of sPIF, we employed two in vitro systems: mouse $\mathrm{C} 2 \mathrm{C} 12$ cells and human myoblasts derived from healthy donors and DMD patients. We first examined the effects of sPIF on myoblast differentiation by analyzing the expression of the myogenic factors,
MyoD and myogenin, which are required for the proper differentiation of myogenic cells during the differentiation and repair of myosin heavy chain $(\mathrm{MyHC})$, which is expressed in differentiated muscle cells. The fusion of myoblasts into differentiated myotubes was also determined. Myoblasts were cultured in a medium containing $2 \%$ horse serum for 10 days to allow cell differentiation. sPIF was added to the cells at the beginning of the culture and every 2 days thereafter. Treatment of $\mathrm{C} 2 \mathrm{C} 12$ cells with sPIF increased the expression of MyoD, myogenin, and MyHC (Fig. 1a) as well as cell fusion (Fig. 1b). Similarly, sPIF increased the expression of $\mathrm{MyHC}$ and troponin in human myoblasts derived from both healthy donors and DMD patients (Fig. 1c). The internalization of sPIF in muscle cells was followed using Fluorescein isothiocyanate (FITC)-labeled sPIF and confocal microscopy. As presented in Fig. 1d, sPIF accumulated in the cells within $72 \mathrm{~min}$ of incubation and was localized in the cytoplasm.

Muscles from DMD patients and mdx mice exhibit increased tissue fibrosis that is due, partly, to the increased transdifferentiation of myoblasts into myofibroblasts. Indeed, recent studies from our laboratory demonstrated that muscle cells from DMD patients express increased fibrogenic phenotypes compared to control cells ${ }^{35}$. In contrast to its promoting effect on the myogenic differentiation of DMD myoblasts, sPIF inhibited the fibrogenic differentiation of these cells and decreased the expression of collagen $1 \mathrm{~A} 1$ and $1 \mathrm{~A} 2$ and that of TGF- $\beta$ (Fig. 1e), which plays a major role in fibrogenesis. Similar results were obtained in two additional DMD myoblasts (data not shown). Another important factor that plays a central role in tissue fibrosis is miR- $21^{36}$. We found that human muscle cells from DMD patients expressed higher levels of miR-21 compared with control muscle cells (Fig. 1f). Treatment with sPIF $(300 \mathrm{nM})$ significantly decreased the expression of miR-21 in DMD muscle cells (Fig. 1g).

Silencing of miR-21 in DMD cells inhibited the expression of collagens $1 \mathrm{~A} 1$ and $1 \mathrm{~A} 2$ (Fig. $1 \mathrm{~h}$ ), and the treatment with SPIF induced an additional modest decrease. These effects were repeated in two additional DMD muscle cells. The results suggest that the downregulation of miR-21 expression by sPIF plays a role in its inhibitory effects on the fibrogenic differentiation of these cells and that additional pathways may be also involved.

\section{SPIF increases myoblast differentiation via the induction of IncRNA H19 and miR-675}

To further analyze the mechanisms involved in the effects of sPIF on muscle differentiation, we focused on the H19/miR-675 pathway. Long non-coding RNA (lncRNA) H19 plays an essential role in muscle differentiation and regeneration, and its biological functions are 


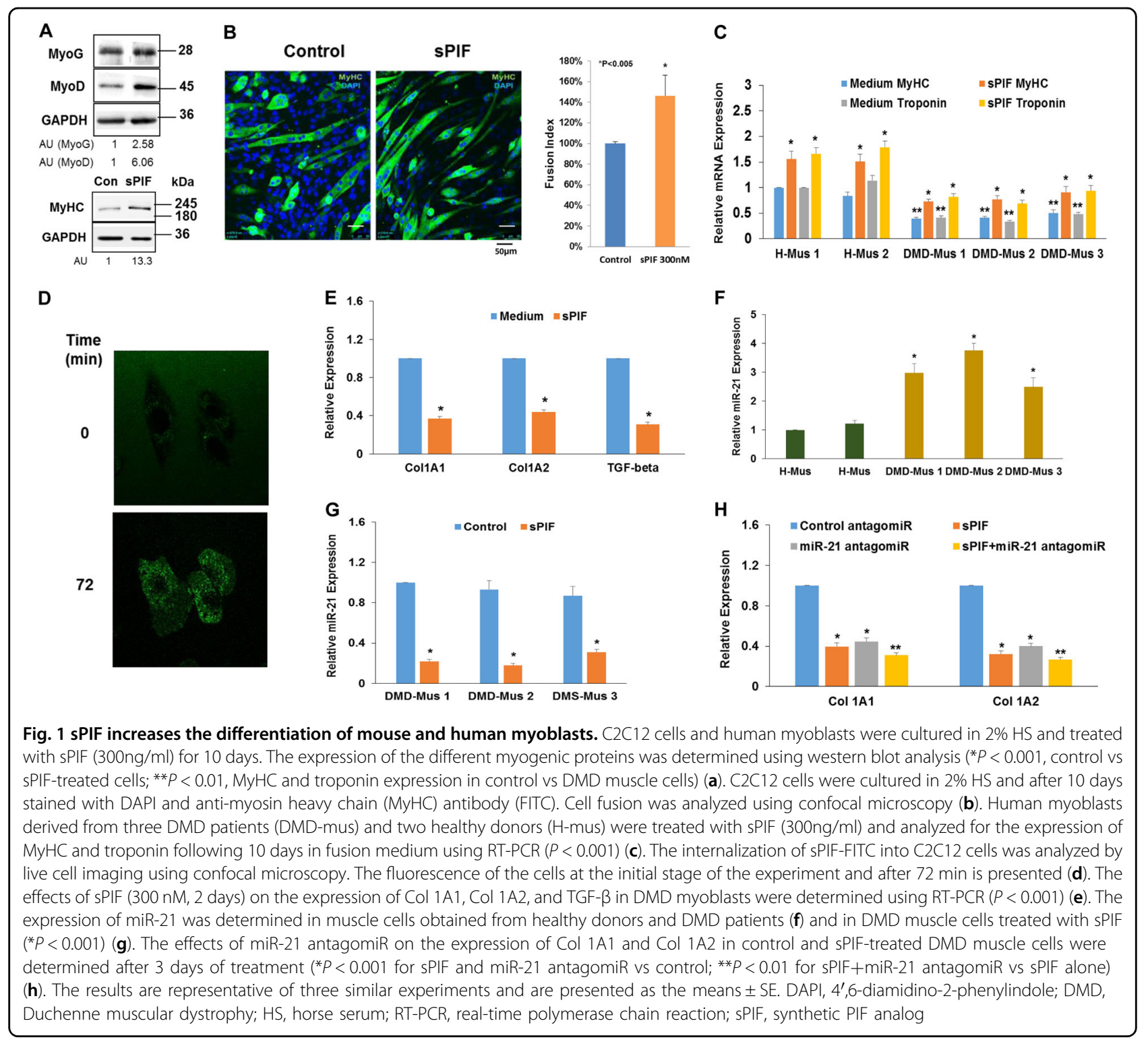

mediated by miR-675-3p and miR-675-5p, which are encoded by exon 1 of $\mathrm{H}_{1} 9^{37}$. Myoblasts derived from DMD patients expressed significantly reduced levels of H19 and miR-675 compared to myoblasts derived from healthy donors (Fig. 2a). The treatment of DMD myoblasts with SPIF significantly upregulated the expression of both H19 (Fig. 2b) and miR-675 (Fig. 2c) in these cells, and these effects were observed in two additional DMD muscle cells (sPIF $100 \mathrm{nM}$; DMD-Mus2-H19: 398.4 \pm 4.2\%; miR-675: $428.96 \pm$ 5.11\%; DMD-Mus3-H19: $312 \pm$ 39.6; miR-675: $287 \pm 23.1 \%$ compared to $100 \%$ in control untreated cells).

To demonstrate that the induction of miR-675 played a role in sPIF-induced myoblast differentiation we performed two experiments. First, we transfected DMD myoblasts with an siRNA duplex that targets H19 and showed that the silencing of H19 decreased miR-675 expression in myoblasts (Fig. 2d) and their differentiation by SPIF (Fig. 2e). We next transfected the DMD myoblasts with miR-675-3p and miR-675-5p mimics and found that the expression of $\mathrm{MyHC}$ and troponin was significantly increased after 5 days of treatment (Fig. 2f). We also examined the effects of SPIF on the differentiation of myoblasts transfected with miR-675 antagomiRs and found that its effects were markedly abrogated, whereas, no significant decrease in the effects of sPIF was observed in myoblasts transfected with a control antagomiR (Fig. 2g). These results indicate that the induction of H19 and the subsequent upregulation of miR-675 mediated, at least partly, some of the increased differentiation of the 


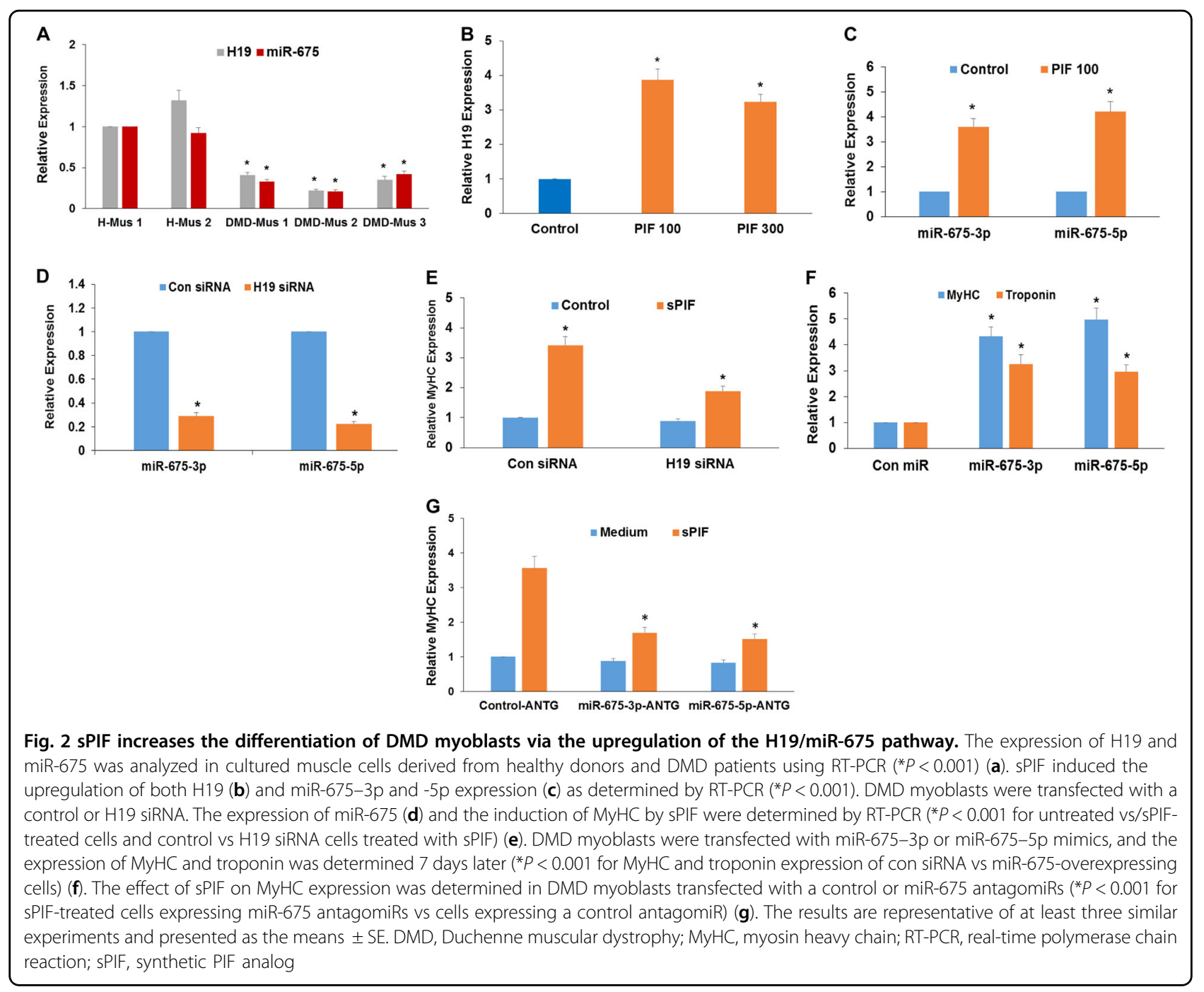

human myoblasts by sPIF. These results exhibited similar patterns in additional DMD muscle cells (data not shown).

\section{sPIF increases utrophin expression via the H19/miR-675/ let-7 pathway}

Utrophin is a functional and structural autosomal paralog of dystrophin. These proteins share binding partners, and upregulating utrophin expression is considered a potential therapeutic approach in DMD. In addition to its effect on myoblast differentiation, sPIF treatment also increased the expression of utrophin in C2C12 (Fig. 3a) and human muscle cells (Fig. 3a, b). This effect of utrophin was observed in additional muscle cultures from healthy and DMD donors (sPIF treated-HMus2: $452.3 \pm 40.2 \%$; DMD-Mus2: $532.8 \pm 48.2 \%$; DMDMus3: $387.1 \pm 30.6 \%$ compared to $100 \%$ in control untreated cells). The induction of utrophin in human myoblasts was partly dependent on the increased expression of H19 since silencing of this lncRNA abrogated the upregulation of utrophin expression (Fig. 3c). H19 acts as a sponge of let- $7^{38}$, which targets the $3^{\prime}$-UTR of utrophin ${ }^{39,40}$, suggesting that the upregulation of H19 may interfere with the ability of let- 7 to target utrophin. We, therefore, examined the effects of let- 7 silencing on utrophin expression in DMD myoblasts and found an increased utrophin expression in the let-7-silenced myoblasts (Fig. 3d). Since sPIF was recently reported to downregulate let-7 expression in neural and lymphoid cells by decreasing its biogenesis, we examined the effects of sPIF on let-7 expression also in muscle cells ${ }^{30}$. Treatment of human myoblasts with sPIF decreased the expression of let-7 (Fig. 3e), and the overexpression of let7 abrogated the upregulation of utrophin by sPIF (Fig. 3f). These results suggest that sPIF may increase utrophin expression by decreasing let-7 expression and by inhibiting the ability of let-7 to target utrophin via the upregulation of H19. 

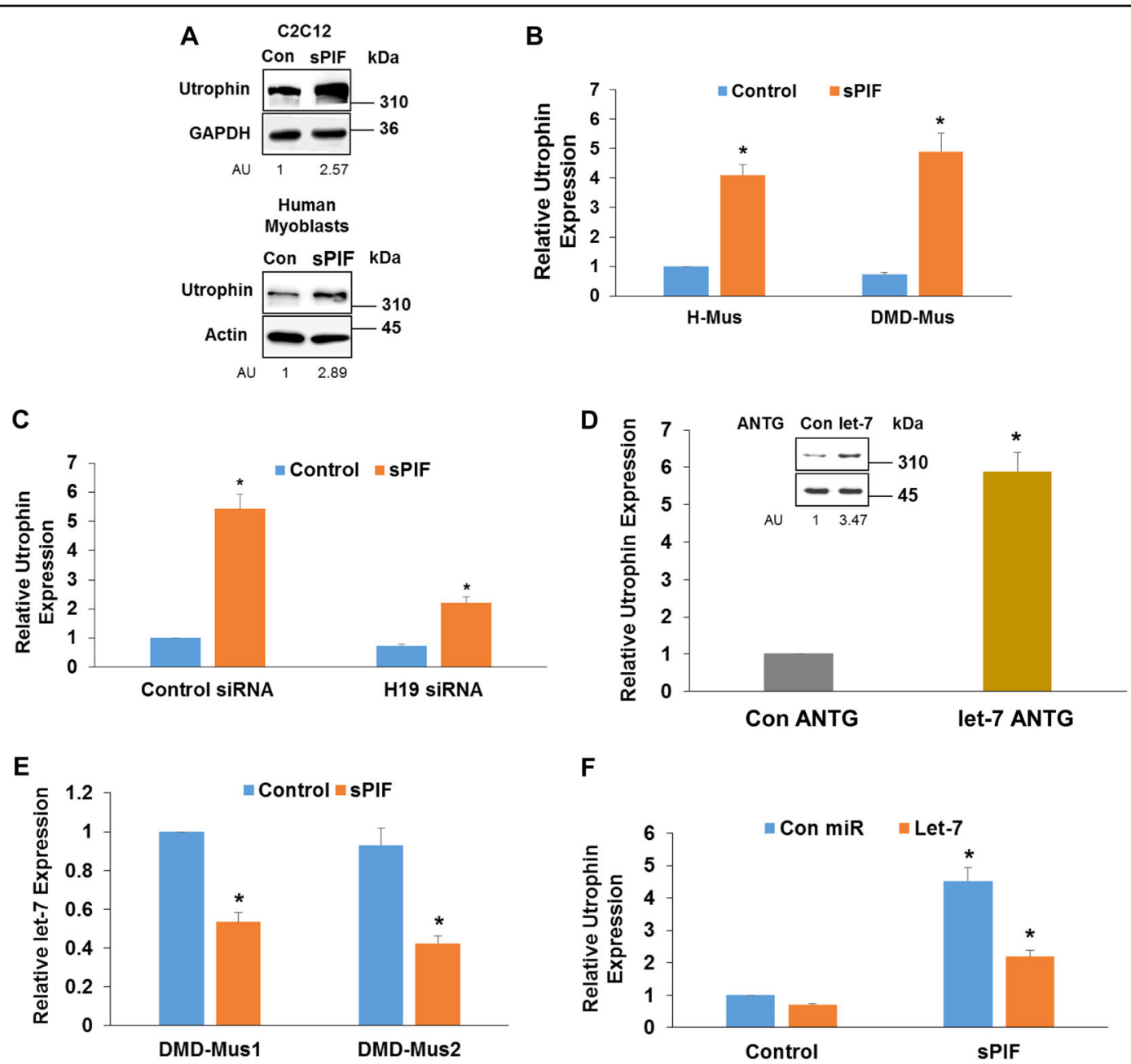

Fig. 3 sPIF upregulates utrophin expression via the H19/Let-7 pathway. C2C12 and human myoblasts were treated with sPIF for 10 days and utrophin expression was determined by western blot analysis (a). Utrophin expression was also analyzed in sPIF-treated DMD myoblasts as determined by RT-PCR (b). DMD myoblasts were silenced for $\mathrm{H} 19$, and the effects of sPIF on utrophin expression were determined following 5 days of treatment (c). DMD myoblasts were treated with a control or let-7 antagomiR, and utrophin expression was analyzed 3days later using RT-PCR and western blot analysis (d). The expression of let-7 was determined in DMD myoblasts following SPIF treatment for 3 days by RT-PCR (e). DMD myoblasts were transfected with a control or let-7 mimic and then treated with SPIF for 5 days. Utrophin expression was determined by RT-PCR (f). The results are representative of at least three similar experiments and presented as the means $\pm \mathrm{SE} .{ }^{*} P<0.001$. DMD, Duchenne muscular dystrophy; RT-PCR, real-time polymerase chain reaction; SPIF, synthetic PIF analog

\section{sPIF decreases CK levels and muscle tissue fibrosis in $\mathrm{mdx}$ mice}

We further studied the therapeutic effects of sPIF on mdx mice that represent a pre-clinical model of DMD and have a premature stop codon mutation on exon 23 of the dystrophin gene, which results in a lack of the mature protein. SPIF $(0.75 \mathrm{mg} / \mathrm{kg})$ was administered twice a day subcutaneously (SC) or SC and intramuscularly to the quadriceps muscles (QC) for 2 weeks. Mice were sacrificed at the end of the treatment and 2 weeks thereafter. We found that sPIF treatment significantly decreased serum CK levels when examined 4 weeks post administration (Fig. 4a), suggesting that sPIF exerted a therapeutic impact in this disease model.
Muscle fibrosis is one of the hallmark processes in DMD, resulting from muscle degeneration and correlating with patient functional deterioration ${ }^{41,42}$. In $\mathrm{mdx}$ mice, fibrosis is mainly observed in the diaphragm and heart ${ }^{43,44}$. We therefore analyzed the effects of sPIF on fibrosis in these tissues. Mice treated with sPIF for 2 weeks were sacrificed 2 weeks later, and the diaphragm tissues were analyzed for collagen I and IV expression using immunofluorescence staining. The expression of collagen I and IV in the diaphragm of sPIF-treated mice was significantly lower compared with that in the tissues of control mice (Fig. 4b). In contrast, sPIF did not induce a significant decrease in collagen I or IV expression in cardiac tissues (data not shown). 

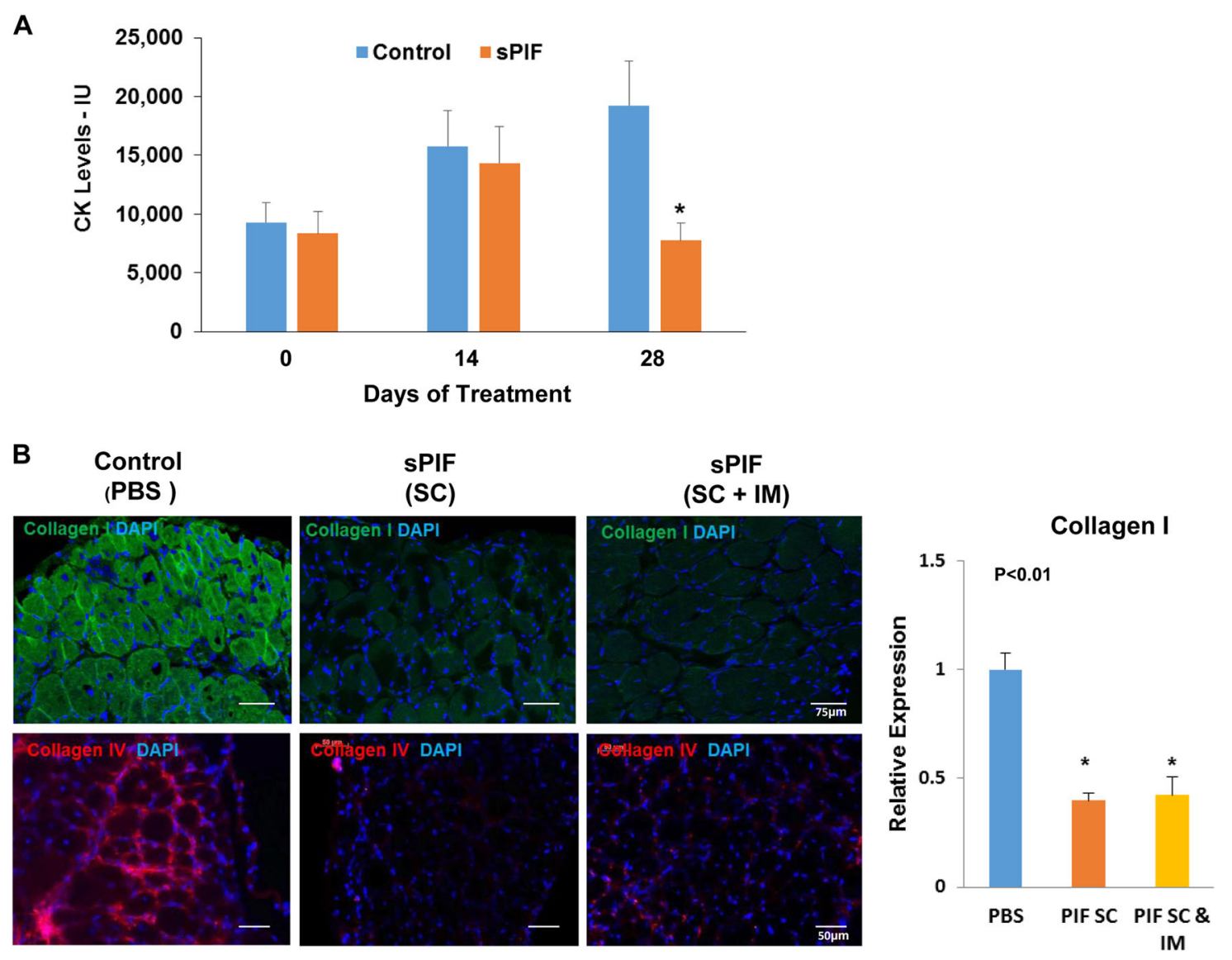

Fig. 4 sPIF decreased CK levels and tissue fibrosis in mdx mice. Mice ( 6 weeks; $n=8$ per group) were injected with PBS (controls) or sPIF ( 0.75 $\mathrm{mg} / \mathrm{kg}$ twice daily for 2 weeks) either SC or once SC and once to the QC muscle. CK levels were analyzed at the end of the treatment and following additional 2 weeks $(* P<0.01)(\mathbf{a})$. Diaphragm muscles from the treated mice were removed and stained for anti-collagen I and collagen IV (b). The levels of collagen I and collagen IV were analyzed for fluorescence signal intensity using CellProfiler software tool and presented as a graph $\left({ }^{*} P<\right.$ 0.01). CK, creatine kinase; PBS, phosphate-buffered saline; QC, quadriceps; SC, subcutaneously; sPIF, synthetic PIF analog

sPIF increases utrophin expression in muscle tissues

Recent studies have reported an inverse correlation between fibrosis and utrophin expression in both patient biopsies and mdx mice ${ }^{44}$. We found that sPIF increased utrophin in cultured cells and therefore examined the effects of sPIF also on utrophin expression in mdx muscle. sPIF treatment significantly increased utrophin expression in the QC (Fig. 5a, b), diaphragm (Fig. 5c), and cardiac (Fig. 5d) muscle tissues of mdx mice.

\section{Discussion}

$\mathrm{DMD}$ is an $\mathrm{X}$-linked muscle degenerative disease that currently has no cure. New therapeutic strategies being pursued for the treatment of this disease include steroids $^{45}$, cell-based therapies ${ }^{46}$, and increasing functional dystrophin expression ${ }^{47}$.

In this study, we focused on SPIF as a potential therapeutic agent for the treatment of DMD and on analyzing the molecular mechanism underlying its effects using both mouse and human in vitro models and mdx mice.
The immune regulatory and regenerative actions of SPIF have been recently reported ${ }^{22,48}$. sPIF promotes embryo differentiation to the blastocyst stage through a targeted action and protects against oxidative stress and protein misfolding ${ }^{15-18}$. The trophic and protective actions of sPIF were also confirmed by demonstrating its effects on reducing inflammation and promoting neural repair and re-myelination in the spinal cord and brain ${ }^{29,32,48}$. Similarly, in a newborn hypoxic ischemic model, sPIF treatment was able to restore the size of the injured cortex ${ }^{30,31}$. sPIF mostly regulates activated immune response without leading to immune suppression and preserves anti-

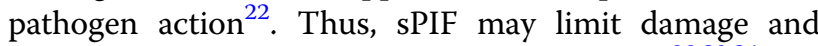
inflammation and promote tissue regeneration ${ }^{28,30,34}$.

sPIF increased the differentiation of both $\mathrm{C} 2 \mathrm{C} 12$ cells and human myoblasts derived from healthy donors and DMD patients, whereas it inhibited the fibrogenic phenotypes of DMD myoblasts. Muscle cells derived from DMD patients exhibited decreased expression of lncRNA H19 and miR-675 compared to healthy donor-derived 


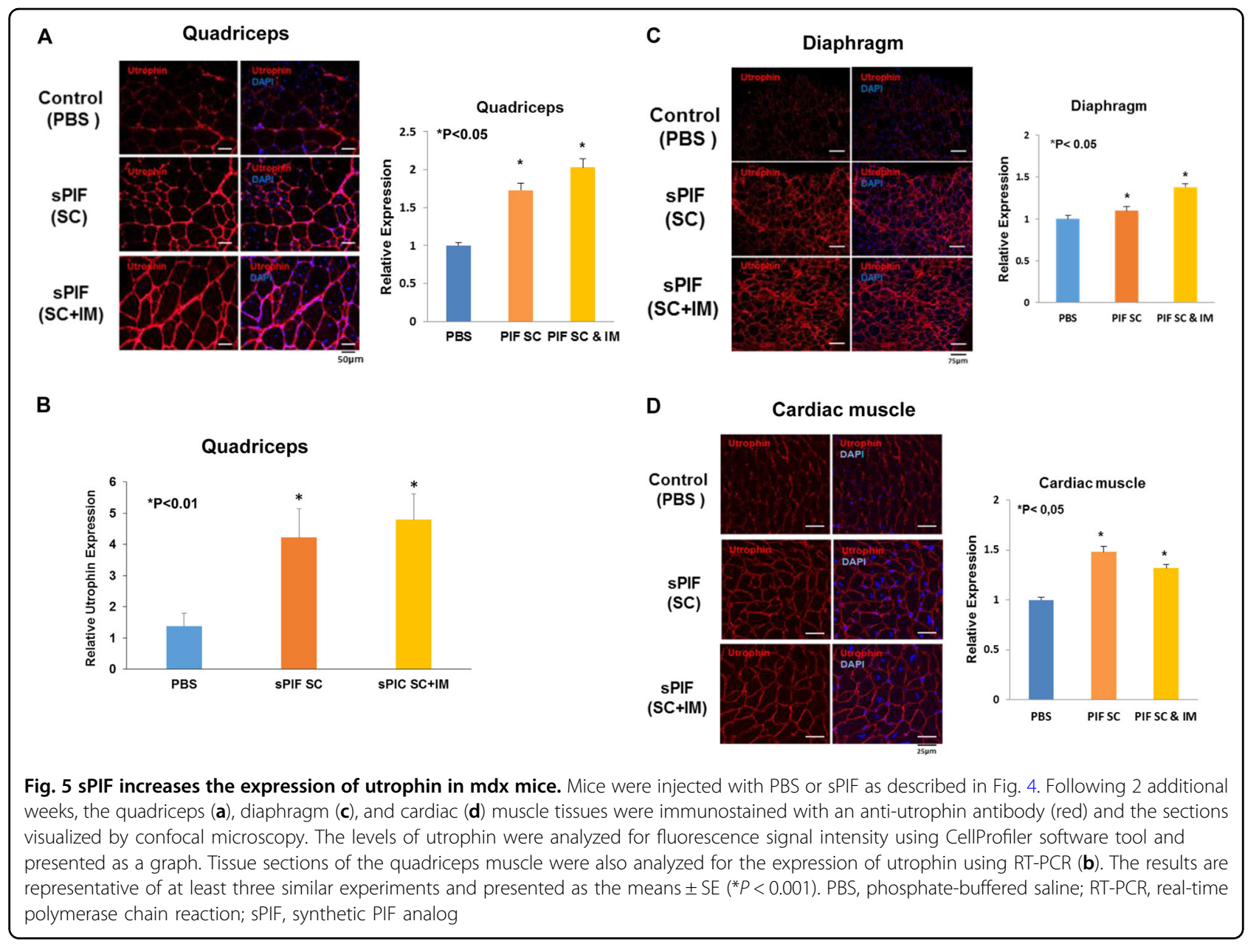

cells. H19 has been identified as an important lncRNA in the function of skeletal muscle differentiation by increasing the expression of miR-675, which in turn inhibits the TGF- $\beta$ pathway ${ }^{37}$. sPIF increased the expression of $\mathrm{H} 19$, miR-675-3p, and miR-675-5p in DMD myoblasts and the differentiation of these cells in an miR-675-dependent manner. This is the first study that demonstrates the effect of SPIF on DMD myoblast differentiation and identifies the H19/miR-675 pathway as a mediator of this effect.

In addition to the in vitro effects, sPIF also exerted a therapeutic impact in mdx mice, which are used as a DMD animal model ${ }^{49}$. Treatment with SPIF significantly decreased the serum CK levels in the treated mice, which reflects the decreased muscle degeneration and tissue damage.

Tissue fibrosis is one of the characteristic pathological hallmarks of DMD and represents a secondary process to the degeneration of muscle tissues ${ }^{41,50}$. Muscle fibrosis is directly correlated with disease progression and poor prognosis $^{51}$. Tissue fibroblasts are considered the main mediators of this process; however, recent studies have reported that DMD myoblasts can also contribute to muscle fibrogenesis ${ }^{50}$. We found that sPIF decreased the fibrosis levels in the diaphragm of the mdx mice as indicated by the decreased immunofluorescence staining of collagen I.

sPIF inhibited the expression of the fibrogenic phenotype and TGF- $\beta$ expression in DMD muscle cells. sPIF also decreased the expression of miR-21, which was higher in DMD muscle cells compared with control myoblasts. miR-21 plays a major role in the fibrosis of various tissues, including liver, renal, and cardiac muscle, and in age-associated muscular dystrophy ${ }^{36,52-54}$. Thus, the upregulation of miR-675 and the decreased miR-21 expression, which target the TGF- $\beta$ pathway and collagen expression, respectively, may mediate the inhibitory effect of SPIF on tissue fibrosis.

Similar to its effects in cultured myoblasts, sPIF also induced the expression of utrophin in the QC, diaphragm, and cardiac tissues of mdx mice. Utrophin is a homolog of dystrophin protein, which is mainly expressed during the embryonic development of skeletal muscles and replaced by dystrophin postnatally ${ }^{55}$. Upregulation of utrophin levels is considered a potential therapeutic approach for 
the treatment of DMD since this protein can substitute for some of the dystrophin functions in the muscles of DMD patients ${ }^{56}$. Recent studies have reported that utrophin is directly targeted by let-7 via binding to its 3'-UTR. Our results demonstrate that sPIF downregulates let-7 expression thereby providing a potential mechanism for the upregulation of utrophin by sPIF. Indeed, sPIF was reported to downregulate let-7 expression also in neuronal and lymphoid cells by inhibiting its biogenesis ${ }^{30}$. Another mechanism by which sPIF increases utrophin levels may be via the upregulation of H19 expression. H19 acts as a sponge of let- 7 and therefore can interfere with the targeting of utrophin by let-7. Interestingly, the expression of utrophin has been reported to be inversely correlated with the level of fibrosis in DMD muscle ${ }^{44}$, which can further contribute to the upregulation of utrophin secondary to the decreased fibrosis induced by sPIF.

The results of this study demonstrate that sPIF promotes muscle differentiation in vitro and exerts a therapeutic impact in mdx mice by decreasing the levels of fibrosis and inducing utrophin expression. The effects of sPIF were at least partially mediated via the upregulation of the H19/miR-675 pathway and downregulation of let-7 and miR-21 (Fig. 6). Additional factors, including inflammatory cytokines and TGF- $\beta$, are also likely to play a role in sPIF effects and are currently being explored. Phase I clinical trial has been now completed for sPIF in autoimmune hepatitis. Based on this and our current results, we propose that sPIF represents a potential therapeutic agent for the treatment of DMD.

\section{Materials and methods Materials}

The following antibodies were employed: myosin heavy chain (sc-376157), myoD (sc-760), Pax-7 (sc-81975), $\beta$-actin HRP-conjugated antibody (sc-47778HRP), and GAPDH (sc-26778) from Santa Cruz Biotechnology

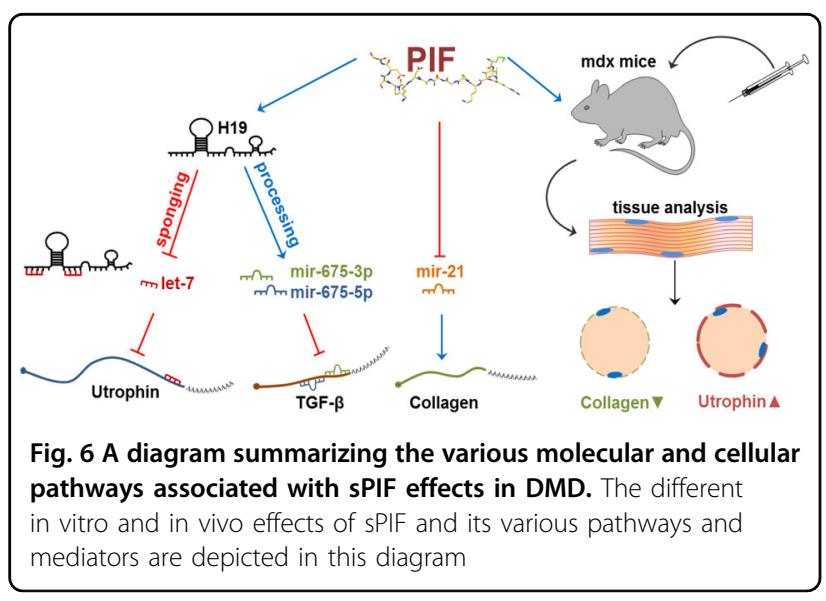

(Dallas, TX, USA); collagen I (Ab21286) and collagen IV (ab19808) from Abcam, Cambridge, UK; utrophin (NCL-DRP2) from Leica Biosystems Inc. (Buffalo Grove, IL, USA); donkey anti-rabbit Cy-3-conjugated antibody (\#711-166-152) and donkey anti-mouse Cy-3conjugated antibody (\#715-165-151) from Jackson ImmunoResearch Laboratories Inc. (West Grove, PA, USA); goat anti-mouse Alexa488-conjugated antibody (\#A-11029) from Molecular Probes Inc., Carlsbad, CA, USA; and 4',6-diamidino-2-phenylindole (DAPI) (D1306) from ThermoFischer Scientific (Oregon City, OR, USA).

\section{Cultures of human myoblasts and $\mathrm{C} 2 \mathrm{C} 12$ cells}

Myoblasts from healthy male donors (H-Mus) and DMD patients (DMD-Mus) were obtained from ScienCell (Carlsbad, CA, USA) and from Creative Bioarray and DV Biologics (Costa Mesa, CA, USA), as previously reported $^{35}$.

C2C12 myoblasts were maintained at subconfluent densities in Dulbecco's modified Eagle's medium (DMEM) supplemented with 10\% fetal bovine serum (Invitrogen, Paisley, UK). Myogenic differentiation was induced by changing subconfluent cells to DMEM containing 2\% heat-inactivated horse serum as recently reported ${ }^{35}$.

\section{Transfection of miRNA mimics, antagomiRs, and shRNAs}

RNA duplexes corresponding to miR-675 and miR-21 mimics were obtained from Sigma (St. Louis, MO, USA). Transfection of the cells with the miRNA duplexes was carried out with siIMPORTER (Millipore) according to the manufacturer's instructions and as previously reported $^{57}$. H19 shRNA was obtained from Dharmacon, and miR-675 and miR-21 antagomiRs were obtained from SBI.

\section{Western blot analysis}

Cell lysates (30 $\mu \mathrm{g}$ protein) were analyzed for the expression of specific markers as previously described ${ }^{35}$.

\section{Fusion analysis}

C2C12 cells were plated in DMEM supplemented with $10 \%$ fetal bovine serum in a $35-\mathrm{mm}$ imaging dish with an ibidi Polymer Coverslip Bottom at high density $(80 \%)$ and allowed to proliferate for $48 \mathrm{~h}$. The medium was then replaced with a differentiation medium containing $2 \%$ horse serum, and the cells were cultured for an additional 10 days. sPIF ( $300 \mathrm{nM}$ ) was added to the culture medium at the beginning of the differentiation phase and every $48 \mathrm{~h}$ thereafter.

Fusion index was quantified as the ratio of DAPIstained nuclei in MyHC-positive multinucleated cells with $\geq 3$ nuclei to the total number of stained nuclei. Analysis was performed using ImageJ software of 49 fields 
in each of the two independent culture dishes per group as previously described ${ }^{35}$.

\section{Real-time quantitative PCR analysis-}

Total RNA was isolated using QIAzol reagent (Qiagen, CA, USA) according to the manufacturer's protocol. Half microgram of RNA was employed to synthesize cDNA by Thermoscript (Invitrogen) with oligo dT primers. mRNAs were analyzed by the TaqMan assay kits for troponin $\mathrm{T}$ (Human TNNT3 Fast skeletal muscle); myosin heavy chain type 2a fast-MyH2 (human: $\mathrm{MyH} 2$ Hs00952980_m1), and utrophin (human: Hs01125975 m1; mouse: Mn01168866_m1) from ThermoFisher Scientific. S12 mRNA was used as an internal control. Expression of miR-21, miR-875, and H19 was determined by the TaqMan stem-loop real-time polymerase chain reaction method. Primers and probes for TaqMan miRNA assay and the endogenous control RNU6B were purchased from System Biosciences.

\section{Animal studies}

All animal experiments were performed in accordance with the guidelines of the Israel Board for Animal Experiments and in compliance with The Israel Animal Welfare Act and Ethics Committee. The mdx mice (6week-old males) were housed in cages under conditions of constant photoperiod (12/12h light/dark) with free access to food and water. Mice were treated with sPIF, $0.75 \mathrm{mg} / \mathrm{kg} / 10 \mu \mathrm{l}$, twice a week for 2 weeks either SC or by a combination of SC and intramuscular injections into the QC muscles. The control mice were injected with saline. The diaphragm, heart, and QC muscles were collected immediately after sPIF administration and for two additional weeks thereafter and were processed for histological and immunofluorescence analyses.

\section{Preparation of sections and immunofluorescence}

Muscle tissues from the control and treated mice were collected and frozen in isopentane cooled with liquid nitrogen. Cryosections were prepared, fixed, and blocked with $0.2 \%(\mathrm{w} / \mathrm{v})$ gelatin and $0.2 \%(\mathrm{v} / \mathrm{v})$ Tween20 (Sigma Aldrich; P1379) in phosphate-buffered saline and immunostained with anti-utrophin antibody (1:50) alone or double-immunostained with collagen type I (1:100) or collagen type IV (1:50) antibodies. Cell nuclei were stained with DAPI (1:1000). As secondary antibodies, goat anti-mouse IgG antibodies with Alexa Fluor dye were used for collagen type I detection and Cy3 anti-goat for utrophin detection. Microscopic observations and image acquisition were performed with Leica SP8 Confocal and Zeiss Axioimager fluorescent microscopes and analyzed using CellProfiler software as previously reported ${ }^{35}$. Images from 8-12 different fields were acquired and analyzed for each mice.

\section{Statistical analysis}

Quantitative measurements are presented as the mean values $\pm \mathrm{SE}$ and visualized in bar charts. Data were analyzed using ANOVA or Student's $t$ test, with correction for datasets with unequal variances.

\section{Acknowledgements}

This study was supported in part by the Association Duchenne Israel, the Israeli Parents' Duchenne/Becker Association, Life for Alon Foundation, Little Step Foundation, Action in Science, and Biolncept.

\section{Author details}

${ }^{1}$ The Mina and Everard Goodman Faculty of Life Sciences, Bar-Ilan University, Ramat-Gan, Israel. ²Department of Neurosurgery, Henry Ford Hospital, Detroit, MI, USA. ${ }^{3}$ Department of Neurology, Talpiot Medical Leadership Program, Chaim Sheba Medical Center, Ramat-Gan, and Sackler Faculty of Medicine, Tel Aviv University, Tel Aviv, Israel. " Biolncept, LLC, New York, NY 10016, USA

\section{Conflict of interest}

Eytan R. Barnea is the Chief Scientific Officer of Biolncept, LLC. Chaya Brodie received a grant from Biolncept, $L L C$. The other authors declare no conflict of interest.

\section{Publisher's note}

Springer Nature remains neutral with regard to jurisdictional claims in published maps and institutional affiliations.

Received: 14 June 2018 Revised: 7 December 2018 Accepted: 17 December 2018

Published online: 28 January 2019

\section{References}

1. Emery, A. E. The muscular dystrophies. Lancet 359, 687-695 (2002).

2. Bushby, K. et al. Diagnosis and management of Duchenne muscular dystrophy, part 1: diagnosis, and pharmacological and psychosocial management. Lancet Neurol. 9, 177-189 (2010).

3. Bushby, K. M. et al. Variability in clinical, genetic and protein abnormalities in manifesting carriers of Duchenne and Becker muscular dystrophy. Neuromuscul. Disord. 3, 57-64 (1993).

4. Blake, D. J., Weir, A., Newey, S. E. \& Davies, K. E. Function and genetics of dystrophin and dystrophin-related proteins in muscle. Physiol. Rev. 82, 291-329 (2002).

5. Keefe, A. C. \& Kardon, G. A new role for dystrophin in muscle stem cells. Nat. Med. 21, 1391-1393 (2015).

6. Bentzinger, C. F., Wang, Y. X. \& Rudnicki, M. A. Building muscle: molecular regulation of myogenesis. Cold Spring Harb. Perspect. Biol. 4, 1-16 (2012).

7. Dumont, N. A. et al. Dystrophin expression in muscle stem cells regulates their polarity and asymmetric division. Nat. Med. 21, 1455-1463 (2015).

8. Uezumi, A. et al. Fibrosis and adipogenesis originate from a common mesenchymal progenitor in skeletal muscle. J. Cell. Sci. 124, 3654-3664 (2011).

9. Bogdanovich, S. et al. Functional improvement of dystrophic muscle by myostatin blockade. Nature 420, 418-421 (2002).

10. Heier, C. R. et al. VBP15, a novel anti-inflammatory and membrane-stabilizer, improves muscular dystrophy without side effects. EMBO Mol. Med. 5, 1569-1585 (2013)

11. Huebner, K. D., Jassal, D. S., Halevy, O., Pines, M. \& Anderson, J. E. Functional resolution of fibrosis in mdx mouse dystrophic heart and skeletal muscle by halofuginone. Am. J. Physiol. Heart Circ. Physiol. 294, H1550-H1561 (2008).

12. Aoki, Y. et al. Bodywide skipping of exons $45-55$ in dystrophic mdx52 mice by systemic antisense delivery. Proc. Natl. Acad. Sci. USA. 109, 13763-13768 (2012).

13. Aartsma-Rus, A. et al. Development of exon skipping therapies for Duchenne muscular dystrophy: A critical review and a perspective on the outstanding issues. Nucleic Acid. Ther. 27, 251-259 (2017).

14. Griggs, R. C. et al. Efficacy and safety of deflazacort vs prednisone and placebo for Duchenne muscular dystrophy. Neurology 87, 2123-2131 (2016). 
15. Stamatkin, C. W. et al. Prelmplantation Factor (PIF) correlates with early mammalian embryo development-bovine and murine models. Reprod. Biol. Endocrinol. 9, 63-74 (2011).

16. Stamatkin, C. W. et al. Preimplantation factor negates embryo toxicity and promotes embryo development in culture. Reprod. Biomed. Online 23, 517-524 (2011).

17. Goodale, L. F. et al. Prelmplantation factor (PIF) protects cultured embryos against oxidative stress: relevance for recurrent pregnancy loss (RPL) therapy. Oncotarget 8, 32419-32432 (2017).

18. Barnea, E. R. et al. Insight into Prelmplantation Factor (PIF*) mechanism for embryo protection and development: Target oxidative stress and protein misfolding (PDI and HSP) through essential RIPK binding site. PLoS One 9 e100263 (2014).

19. Simone, N. Di et al. Synthetic Prelmplantation Factor (PIF) prevents fetal loss by modulating LPS induced inflammatory response. PLoS One 12, e0180642 (2017).

20. Moindjie, $\mathrm{H}$. et al. Preimplantation factor is an anti-apoptotic effector in human trophoblasts involving p53 signaling pathway. Cell Death Dis. 7, e2504 (2016).

21. Paidas, M. J. et al. A genomic and proteomic investigation of the impact of preimplantation factor on human decidual cells. Am. J. Obstet. Gynecol. 202 459.e1-8 (2010)

22. Barnea, E. R. et al. Prelmplantation Factor (PIF) orchestrates systemic antiinflammatory response by immune cells: effect on peripheral blood mononuclear cells. Am. J. Obstet. Gynecol. 207, 313. e1-313. e11 (2012).

23. Barnea, E. R. et al. PIF direct immune regulation: Blocks mitogen-activated PBMCs proliferation, promotes $\mathrm{TH} 2 / \mathrm{TH} 1$ bias, independent of $\mathrm{Ca} 2+$. Immunobiology 220, 865-875 (2015).

24. Barnea, E. R. et al. Prelmplantation factor (PIF) regulates systemic immunity and targets protective regulatory and cytoskeleton proteins. Immunobiology $\mathbf{2 2 1}$ 778-793 (2016).

25. Roussev, R. G. et al. Preimplantation factor inhibits circulating natural killer cell cytotoxicity and reduces CD69 expression: Implications for recurrent pregnancy loss therapy. Reprod. Biomed. Online 26, 79-87 (2013).

26. Weiss, L. et al. Preimplantation factor (PIF) analog prevents type I diabetes mellitus (TIDM) development by preserving pancreatic function in NOD mice Endocrine 40, 41 (2011).

27. Chen, Y. C. et al. Preimplantation factor prevents atherosclerosis via its immunomodulatory effects without affecting serum lipids. Thromb. Haemost. 115, 1010-1024 (2016).

28. Shainer, R. et al. Prelmplantation factor (PIF) therapy provides comprehensive protection against radiation induced pathologies. Oncotarget 7, 58975-58994 (2016).

29. Weiss, L. et al. Preimplantation Factor $\left(\mathrm{PIF}^{*}\right)$ reverses neuroinflammation while promoting neural repair in EAE model. J. Neurol. Sci. 312, 146-157 (2012).

30. Mueller, M. et al. Prelmplantation factor promotes neuroprotection by targeting microRNA let-7. Proc. Natl. Acad. Sci. USA 111, 13882-13887 (2014).

31. Mueller, M. et al. Prelmplantation Factor bolsters neuroprotection via modulating Protein Kinase A and Protein Kinase C signaling. Cell Death Differ. 22, 2078-2086 (2015).

32. Migliara, G. et al. PIF* promotes brain re-myelination locally while regulating systemic inflammation-clinically relevant multiple sclerosis $M$. smegmatis model. Oncotarget 8, 21834-21851 (2017)

33. Azar, Y. et al. Prelmplantation Factor reduces graft-versus-host disease by regulating immune response and lowering oxidative stress (Murine Model). Biol. Blood. Marrow Transplant. 19, 519-528 (2013).

34. Feichtinger, M., Barnea, E. R., Nyachieo, A., Brännström, M. \& Kim, S. S. Allogeneic ovarian transplantation using immunomodulator preimplantation factor (PIF) as monotherapy restored ovarian function in olive baboon. J. Assist. Reprod. Genet. 35, 81-89 (2017).
35. Bier, A. et al. Placenta-derived mesenchymal stromal cells and their exosomes exert therapeutic effects in Duchenne muscular dystrophy. Biomaterials 174, 67-78 (2018).

36. Huang, Y., He, Y. \& Li, J. MicroRNA-21: A central regulator of fibrotic diseases via various targets. Curr. Pharm. Des. 21, 2236-2242 (2015).

37. Dey, B. K., Pfeifer, K. \& Dutta, A. The H19 long noncoding RNA gives rise to microRNAs miR-675-3p and miR-675-5p to promote skeletal muscle differentiation and regeneration. Genes Dev. 28, 491-501 (2014).

38. Kallen, A. N. et al. The imprinted H19 IncRNA antagonizes Let-7 microRNAs. Mol. Cell 52, 101-112 (2013).

39. Basu, U. et al. Translational regulation of utrophin by miRNAs. PLoS One 6 e29376 (2011).

40. Mishra, M. K., Loro, E., Sengupta, K., Wilton, S. D. \& Khurana, T. S. Functional improvement of dystrophic muscle by repression of utrophin: Let-7c interaction. PLoS One 12, e0182676 (2017).

41. Mann, C. J. et al. Aberrant repair and fibrosis development in skeletal muscle. Skelet. Muscle 1, 21 (2011).

42. Li, Y. et al. Transforming growth factor-beta1 induces the differentiation of myogenic cells into fibrotic cells in injured skeletal muscle: a key event in muscle fibrogenesis. Am. J. Pathol. 164, 1007-1019 (2004).

43. Barbin, I. C. et al. Diaphragm degeneration and cardiac structure in $m d x$ mouse: potential clinical implications for Duchenne muscular dystrophy. J. Anat. 228, 784-791 (2016).

44. Levi, O., Genin, O., Angelini, C., Halevy, O. \& Pines, M. Inhibition of muscle fibrosis results in increases in both utrophin levels and the number of revertant myofibers in Duchenne muscular dystrophy. Oncotarget $\mathbf{6}$ 23249-23260 (2015).

45. Tidball, J. G. \& Wehling-Henricks, M. Evolving therapeutic strategies for Duchenne muscular dystrophy: targeting downstream events. Pediatr. Res. 56, 831-841 (2004).

46. Farini, A., Razini, P., Erratico, S., Torrente, Y. \& Meregalli, M. Cell based therapy for duchenne muscular dystrophy. J. Cell. Physiol. 221, 526-534 (2009).

47. Hoffman, E. P. et al. Restoring dystrophin expression in Duchenne muscular dystrophy muscle. Am. J. Pathol. 179, 12-22 (2011).

48. Barnea, E. R. et al. Immune regulatory and neuroprotective properties of preimplantation factor: From newborn to adult. Pharmacol. Ther. 156, 10-25 (2015).

49. Grounds, M. D., Radley, H. G., Lynch, G. S., Nagaraju, K. \& De Luca, A. Towards developing standard operating procedures for pre-clinical testing in the $\mathrm{mdx}$ mouse model of Duchenne muscular dystrophy. Neurobiol. Dis. 31, 1-19 (2008).

50. Wynn, T. Cellular and molecular mechanisms of fibrosis. J. Pathol. 214 199-210 (2008).

51. Desguerre, I. et al. Endomysial fibrosis in Duchenne muscular dystrophy: a marker of poor outcome associated with macrophage alternative activation. J. Neuropathol. Exp. Neurol. 68, 762-773 (2009).

52. Ardite, E. et al. PAl-1-regulated miR-21 defines a novel age-associated fibrogenic pathway in muscular dystrophy. J. Cell. Biol. 196, 163-175 (2012).

53. Reddy, S. et al. miR-21 is associated with fibrosis and right ventricular failure. JCl Insight 2, e91625 (2017).

54. Liu, Y. et al. TGF- $\beta 1$ promotes scar fibroblasts proliferation and transdifferentiation via up-regulating MicroRNA-21. Sci. Rep. 6, 32231 (2016).

55. Miura, P. \& Jasmin, B. J. Utrophin upregulation for treating Duchenne or Becker muscular dystrophy: how close are we? Trends Mol. Med. 12, 122-129 (2003).

56. van Deutekom, J. C. \& van Ommen, G. J. Advances in Duchenne muscular dystrophy gene therapy. Nat. Rev. Genet. 4, 774-783 (2003).

57. Bier, A. et al. MicroRNA-137 is downregulated in glioblastoma and inhibits the stemness of glioma stem cells by targeting RTVP-1. Oncotarget 4, 665-676 (2013). 